\title{
Modeling and experimental validation of an immersed thermo-mechanical part-scale analysis for laser powder bed fusion processes
}

\author{
Massimo Carraturo ${ }^{\mathrm{a}, *},{\text { John } \text { Jomo }^{\mathrm{b}} \text {, Stefan Kollmannsberger }}^{\mathrm{c}}$, Ernst Rank ${ }^{\mathrm{b}}$, \\ Alessandro Reali ${ }^{\text {a }}$, Ferdinando Auricchio ${ }^{\text {a }}$ \\ ${ }^{a}$ Department of Civil Engineering and Architecture, University of Pavia, via Ferrata 3, \\ 27100 Pavia, Italy \\ ${ }^{b}$ Chair for Computation in Engineering, Technische Universität München, Arcisstr. 21, \\ 80333 München, Germany \\ ${ }^{c}$ Chair of Computational Modeling and Simulation, Technische Universität München, \\ Arcisstr. 21, 80333 München, Germany
}

\begin{abstract}
The capability of correctly predicting part deflections after support removal is important to asses the quality of a final artifact produced by laser powder bed fusion (LPBF) technology. The finite element method is usually employed to perform part-scale thermo-mechanical analysis to estimate the final distortion of $3 \mathrm{D}$ printed parts. Due to the high flexibility of LPBF additive manufacturing, most of the components produced by means of such a technology have an optimized shape and complex geometrical features. Consequently, the process of generating an analysis suitable mesh starting from the original 3D virtual model turns out to be a non-trivial task. Immersed boundary methods represent a possible solution to perform accurate process simulation without the meshing burden. In this work, an immersed numerical framework to perform thermo-mechanical part-scale analysis is experimentally validated by means of part deflection measurements obtained for a single-cantilever structure after support removal. The comparison between simulation and experiment shows that the proposed numerical framework is able to deliver results with an almost perfect correlation to the measured data and a maximum relative error below
\end{abstract}

\footnotetext{
* Corresponding author

Email address: massimo.carraturo@unipv.it (Massimo Carraturo)
} 
$5 \%$.

Keywords: Laser powder bed fusion, Finite Cell Method, Experimental validation, Part-scale model

\section{Introduction}

Laser powder bed fusion (LPBF), alternatively known as selective laser melting (SLM), selective laser sintering (SLS), electron beam melting (EBM), direct metal laser sintering (DMLS), or direct metal laser melting (DMLM), are additive manufacturing (AM) processes consisting of either a laser or an electron beam which selectively melts a layer of metal powder, building the final product by means of a layer-by-layer process. Nowadays, most of the metal additive manufacturing systems employ laser powder bed fusion technology due to its capacity to produce small geometrical features, to cover a larger range of scales, to deliver more accurate surface finishing, to achieve lower porosity, and to obtain better mechanical properties of the resulting part compared to other AM technologies [1. Furthermore, compared to more traditional manufacturing techniques, LPBF technology allows to abolish most of the standard production constraints, opening the possibility to optimally design components and to manufacture complex shapes. Due to these key features, LPBF AM has seen a rapid growth over the last decades.

Nevertheless, there are still open issues that do not allow us to consider LPBF a "mature" technology yet. Currently, one of the main limitations of LPBF processes is the difficulty to predict a-priori the final shape of the printed artifact. In fact, during an LPBF process a single material point undergoes multiple, rapid melting-solidification cycles, which induce high residual stresses within the component. These residual stresses are then partially released once the part is removed from its support, leading to a deformed component.

Numerical analysis can play an important role enabling the prediction of the final deformation of a printed component without undergoing long and expensive trial and error procedures. However, due to its multi-scale nature in both space 
and time, numerical simulations of LPBF processes are extremely challenging from a modeling as well as from a numerical point of view. For detailed reviews on the state of the art of LPBF AM process simulations we refer to [2-4]. In particular, we can distinguish three main groups of physical models which differ in the spatial and temporal scale they aim at solving [5]: powder, micro-structural, and part-scale models. Powder models aim at predicting melt-pool geometries and thermal profiles starting from powder and solid material properties as well as process parameters [6, 7]; the required high spatio-temporal resolution limits this kind of analysis either to a single or to few adjacent laser tracks. Microstructural models focus on the grain growth process occurring during the rapid melting-solidification cycles in very localized regions, often limiting the analysis to 2D-settings [8-10]. The objective of part-scale models is instead to deliver an accurate prediction of part deflections and residual stresses at large-scale.

Most of the numerical methods proposed in the literature for part-scale AM simulations employ the finite element method (FEM) to perform a coupled thermo-mechanical analysis of the LPBF AM process. This kind of analysis can be computationally very expensive due to the long time-scale of the process and the non-linear, plastic behavior of the material. Therefore, most of the works consider direct energy deposition (DED) processes, since they usually involve a lower range of spatial and temporal scales compared to LPBF [11-14].

Only recently, physical and numerical models have been developed to predict part distortions and residual stresses for LPBF AM processes. For instance, a full thermo-mechanical simulation based on a multi-scale approach is carried out by Gouge et al. [15] for LPBF processes of complex parts using Pan Solver from Autodesk Inc. In Liang et al. [16] the modified inherent strain method [17] is applied to predict distortions of 3D printed components manufactured by means of LPBF technology. Both these approaches employ inherent strains evaluated with thermo-mechanical high-fidelity simulations which resolve the melt-pool length-scale on a small reference domain.

A simple yet effective method is the so-called pragmatic approach introduced by Williams et al. [18, where - without involving any inherent strain evalua- 
tion - a weakly-coupled thermo-mechanical problem is solved at the scale of a complete component. This method sequentially activates deposited layers at melting temperature. Although this represents only a rough homogenization of the microscopic stress states induced by the individual laser tracks, it was demonstrated that such a model may predict deflections of the final artifact with deviations from the experimental measurements in the range of $5-10 \%$. In the present work, we employ a similar physical model, following an extension of the pragmatic approach as proposed in [19].

As all standard FEM analyses, also the aforementioned FEM-based approaches require a computational mesh which conforms to the physical boundary of the artifact to be simulated. Generating such a mesh can be a time-consuming task for the complex geometries typically involved in AM production. Moreover, the 3D virtual model (usually stored in .stl file format) might present geometric flaws which have to be repaired before mesh generation, and the generated mesh may be over-refined in parts of the domain where critical geometrical features (e.g., sharp corners, small holes, etc.) are presents. We finally remark that such a mesh must not only conform to the geometry but also to the layers of the built process.

Despite all the issues stated above, the problem of converting raw .stl data into an analysis-suitable mesh is generally neglected in the literature of simulation for AM. In the opinion of the authors, this step is instead crucial since one of the key features of this technology is the possibility to produce optimized and complex topological parts which are not trivial to be meshed. Therefore, in industrial practice, conversion from CAD to analysis-suitable meshes turns out to be critical when performing numerical simulations of AM processes [20].

Immersed boundary methods can be an attractive alternative to effectively handle the numerical analysis of complex geometrical components. Even if most of the modern CAD software can directly generate "water-tight" models, for such a kind of complex geometries an .stl check is usually recommended before the build is performed [21]. The .stl check usually includes a watertight check, a check of normal vectors orientation, and redundant or duplicated elements 
removal.

Employing standard numerical methods, the design and analysis process consists of the following steps:

1. Generate a $3 \mathrm{D}$ virtual model in a $\mathrm{CAD}$ environment;

2. Check-and-repair the .stl file using an .stl file repair software (e.g., MAGICS $\left.{ }^{\circledR}\right)$;

3. Generate an analysis suitable mesh;

4. Run the thermo-mechanical simulation using a FEM code.

With such a workflow, if, for instance, the numerical results show that the final deflection of the part is too large, the CAD model must be modified and the overall procedure entirely repeated, whereas, adopting an immersed approach would avoid step 2 and 3, running the thermo-mechanical analysis directly on the CAD model. In such a way, the immersed workflow will require an stl repair software only once the final design is ready to be printed.

In particular, the Finite Cell Method (FCM) [22] has already been applied to simulate thermal [23] and thermo-mechanical [24] problems, but limited to the problem of the identification of melt-pool length-scales. In this work, FCM is applied to simulate an LPBF process at part-scale by means of a layer-by-layer activation process. Numerical results are validated by means of the publicly available experimental measurements provided by the US National Institute of Standards and Technology (NIST) for the part deflection of a single-cantilever structure of Inconel 625 (IN625 ${ }^{\circledR}$ ).

All numerical results presented in this work are obtained using AdhoC++, a high-order finite element code implementing FCM, that is developed and maintained at the Chair for Computation in Engineering at the Technical University of Munich.

The outline of this paper is as follows. In Section 2, we present the set of governing equations used to describe the weakly-coupled thermo-mechanical problem. Section 3 briefly describes the setup of experimental measurements obtained at NIST. In Section 4 we first shortly recall the main ideas underlying 
FCM and then detail its implementation to solve part-scale LPBF AM processes.

In Section 5 we present and discuss our numerical results, comparing them with the experimental measurements, while we draw conclusions and provide an outlook in Section 6 .

\section{Governing equations}

In the present work a weakly coupled thermo-mechanical analysis is performed in a Langrangian reference frame to model an LPBF manufacturing process. Interested readers are referred to [25-27] for further details on thermomechanical finite element modeling for both welding and AM.

\subsection{Thermal problem}

Assuming the material obeys Fourier's law of heat conduction, to model the LPBF thermal process we employ the heat transfer diffusion equation defined as follows:

$$
\rho C_{p} \frac{\partial T}{\partial t}-\nabla \cdot(k \nabla T)=Q
$$

where $\rho$ is the temperature-dependent density of the material, $C_{p}$ is the temperature-dependent specific heat capacity, $k$ is the temperature-dependent thermal conductivity, $T$ indicates the temperature field, $t$ the time, and $Q$ the equivalent heat source evaluated as:

$$
Q=\frac{\eta P}{\mathrm{HAV}}
$$

with $\eta$ the absorptivity of the material, $P$ the laser power, and HAV the heat affected volume, i.e., the volume where the equivalent heat source is applied. Since the phase-change transformation occurs within a very short time interval, the latent heat term associated to the material phase-change can be neglected in part-scale thermal models, as demonstrated in [28].

In our model, we assume that the heat is dissipated by conduction through the powder surrounding the solidified domain as well as by radiation and convection through the powder layer upper surface. The former is modeled using 
a powder conduction heat loss term $q^{\text {pow }}$, defined as follows:

$$
q^{\text {pow }}=h^{\text {pow }}\left(T-T_{e}\right)
$$

where $T_{e}$ is the temperature of the surrounding environment and $h^{\text {pow }}$ is the heat transfer coefficient (HTC) by conduction through the powder. Following [28] our numerical model defines both heat convection and radiation by means of a single convective heat loss term $q^{\text {loss }}$, defined as:

$$
q^{l o s s}=h^{l o s s}\left(T-T_{e}\right)
$$

where $h^{\text {loss }}$ is the corresponding equivalent HTC. This simplified assumption is justified by Chiumenti et al. 28] since at part-scale it is difficult to distinguish between the two heat transfer modes. In this work we assume a HTC by conduction $h^{\text {pow }}=0.1\left[\mathrm{~W} / \mathrm{K} / \mathrm{m}^{2}\right]$ and an equivalent HTC by radiation and convection $h^{\text {loss }}=0.5\left[\mathrm{~W} / \mathrm{K} / \mathrm{m}^{2}\right]$.

\subsection{Mechanical problem}

The mechanical response of the component is calculated using a thermal load derived from the solution of the thermal problem. The mechanical equilibrium equation is given by:

$$
\nabla \cdot \boldsymbol{\sigma}=\mathbf{0}
$$

where $\boldsymbol{\sigma}$ is the second-order stress tensor defined as:

$$
\boldsymbol{\sigma}=\mathbf{D}^{e} \varepsilon^{e}
$$

with $\mathbf{D}^{e}$ the fourth-order material tensor and $\varepsilon^{e}$ the elastic strain tensor. The total strain $\varepsilon$ can be split into three components:

$$
\varepsilon=\varepsilon^{t h}+\varepsilon^{e}+\varepsilon^{p}
$$


with $\varepsilon^{t h}$ and $\varepsilon^{p}$ the thermal and plastic strains, respectively. The thermal strain drives the mechanical problem and acts as an external (thermal) load that can be evaluated as:

$$
\varepsilon^{t h}=\alpha \Delta T \mathbf{I}
$$

where $\alpha$ is the temperature dependent thermal expansion coefficient and $\mathbf{I}$ the second-order identity tensor. In this work, we consider small strain thermoelasto-plasticity. Following the von Mises stress criterion together with a Prandtl-Reuss flow rule, we can define the yield function $\Phi$ and the plastic strain rate $\dot{\varepsilon}^{p}$ as follows:

$$
\begin{aligned}
\Phi & =\sigma_{v m}-\sigma_{y}(\gamma) \leq 0, \\
\dot{\boldsymbol{\varepsilon}}^{p} & =\dot{\gamma} \frac{\partial \Phi}{\partial \boldsymbol{\sigma}}
\end{aligned}
$$

where $\sigma_{v m}$ is the equivalent von Mises stress, $\sigma_{y}(\gamma)$ the yield stress with linear isotropic hardening, and $\gamma$ the equivalent plastic strain.

\section{Experimental setup}

To experimentally validate the numerical method presented in this work, we compare the simulated part deflection with experimental data obtained for the AMBench2018 challenge proposed by NIST [29]. All the information regarding the design and the setup of the experiment reported herein are available on the AMBench2018 website [29].

In this section, we provide only a brief description of the experimental setup for the cantilever structure of IN625 described in Figure 1. For this benchmark, two manufacturing processes were performed on two different machines: an Additive Manufacturing Metrology Testbed (the NIST in-house build machine) and an EOS M270. Measurements of part deflection after plate removal are reported only for structures printed on the EOS M270. Therefore in the remaining part of this work we will consider only the EOS M270 setup. 


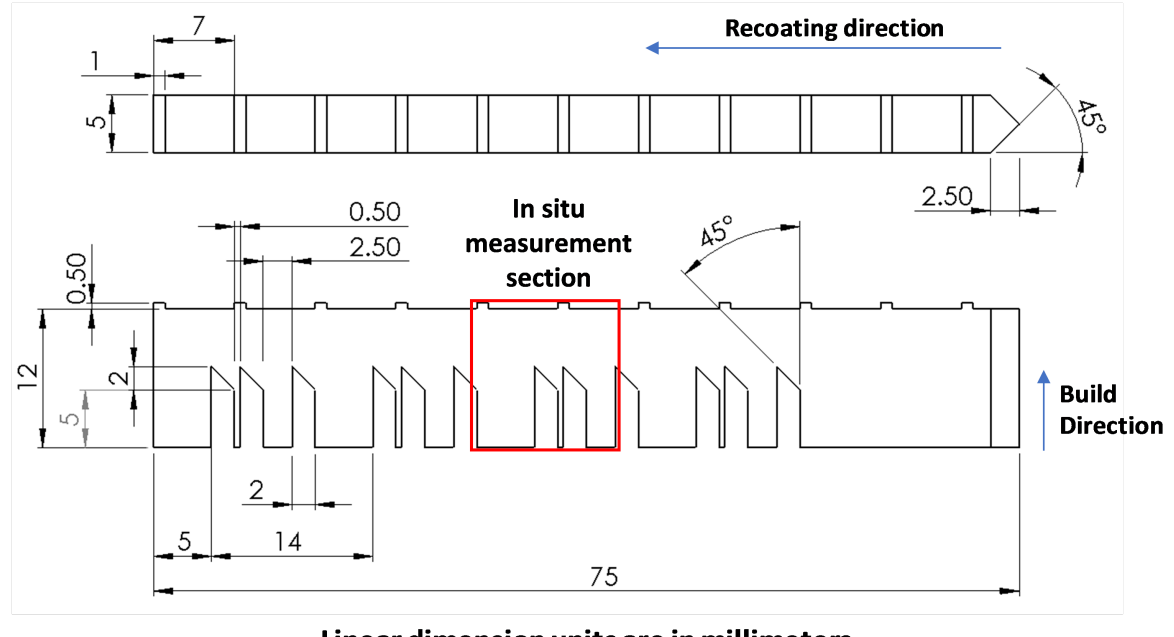

Linear dimension units are in millimeters

Figure 1: Geometry of the build part (source [29]).

As shown in Figure 2 on a single building plate four occurrences of the same geometry were built.

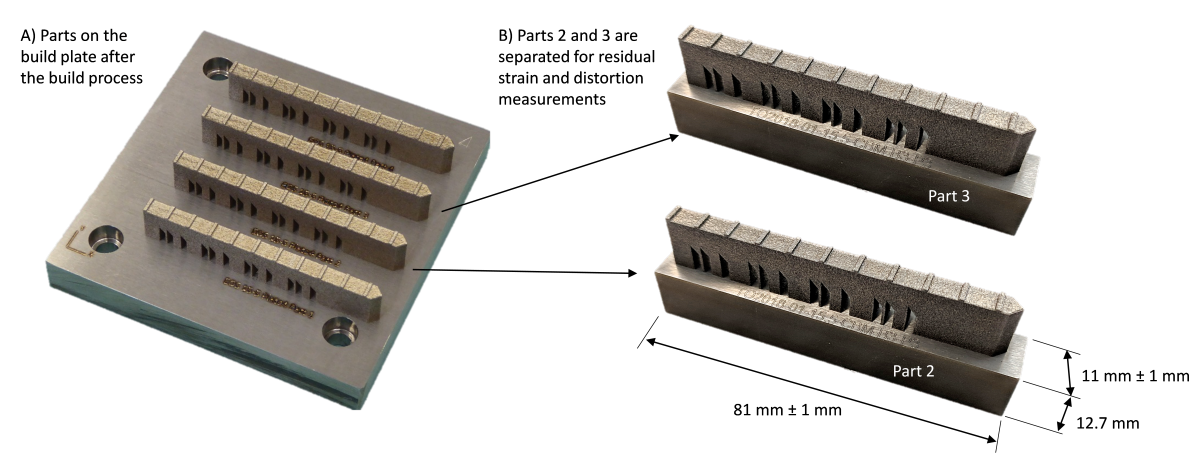

Figure 2: AMB2018-01 building plate in EOS M270 (source 29]).

In our code, we do not model the entire building plate since the four structures have sufficient space between them, thus we can assume no interaction between adjacent structures. For our numerical simulation, we consider a cuboidal substrate having dimensions $90 \mathrm{~mm} \times 6 \mathrm{~mm} \times 12.7 \mathrm{~mm}$, keeping the original substrate height which plays an important role in the solution of the thermal problem acting like a thermal sink. The $x-y$ plane dimensions of the substrate 
are obtained by scaling the outline of the structure geometry by a factor 1.2. We use the same temperature dependent material properties described in Section 4.5 for both the substrate and the structure.

Table 1 reports the process parameters used during the build process for the EOS M270. After building, part of the final structure is removed from the support plate by means of electrical discharge machining (EDM), allowing the part to deflect upward due to the residual stress. The 11 ridges built on the upper surface of the structure (see Figure 3) are ground to obtain a smooth surface allowing an accurate prediction of the deflection by means of a coordinate measuring machine. Different measurements were performed at the NIST laboratories, including upward deflection after removing the part from the substrate. For further details on the measuring process we refer to [30].

In the present work, we aim at replicating the measured upward deflection of the structure by means of the numerical method described in the following section.

\begin{tabular}{ll}
\hline Parameters & Value \\
\hline Total number of layers & 625 \\
Average layer time & $52 \mathrm{~s}$ \\
Layer height & $20 \mu \mathrm{m}$ \\
Contour scan speed & $900 \mathrm{~mm} / \mathrm{s}$ \\
Contour laser power & $100 \mathrm{~W}$ \\
Infill scan speed & $800 \mathrm{~mm} / \mathrm{s}$ \\
Infill laser power & $195 \mathrm{~W}$ \\
Hatch distance & $100 \mu \mathrm{m}$ \\
Laser spot size (FWHM) & $50 \mu \mathrm{m}$ (estimated) \\
\hline
\end{tabular}

Table 1: Process parameters 29

\section{Numerical method}

In this work, we employ the Finite Cell Method, an immersed boundary finite element method, to solve the thermo-mechanical problem defined in Section 2 FCM, thanks to its immersed nature, allows to solve physical problems on complex geometries avoiding time consuming conforming mesh generation 


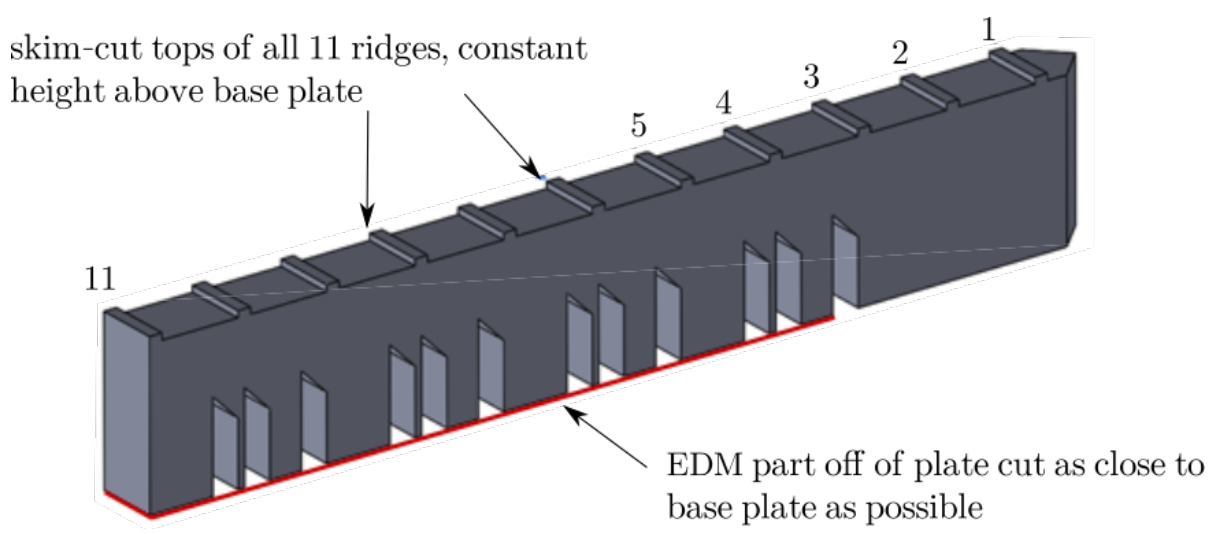

Figure 3: Original .stl file with measurement ridges and removed support description (source 29]).

processes. Further details on the implementation of an FCM-based thermomechanical problem solver can be found in [31].

\subsection{The Finite Cell Method}
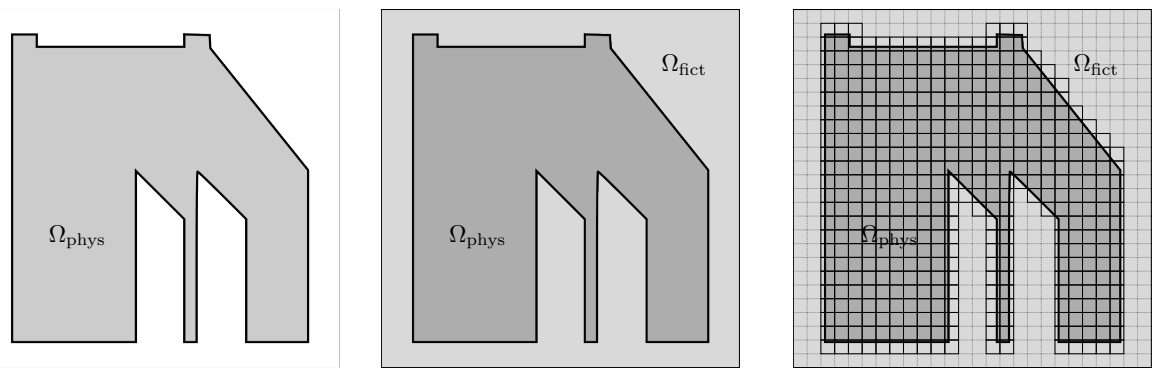

(a) Physical domain $\Omega_{\text {phys }}$.

(b) Fictitious domain extension.

(c) Finite cell mesh.

Figure 4: Illustration of the Finite Cell Method.

Figure 4 depicts the main idea of the Finite Cell Method. Starting from a generic initial domain $\Omega_{\text {phys }}$, referred to as the physical domain, FCM extends $\Omega_{\text {phys }}$ by adding a fictitious domain $\Omega_{\text {fict }}$ such that the final domain $\Omega=\Omega_{\text {phys }} \cup$ $\Omega_{\text {fict }}$ has a simple shape (e.g., a rectangle in two-dimensions or a cuboid in three-dimensions) that can be trivially discretized using regular elements, see, e.g., 22,32$]$. 
Structured Cartesian meshes, whose elements do not conform to the boundary of the physical domain $\Omega_{\text {phys }}$ are commonly used in FCM. These elements are referred to as cells to differentiate them from boundary conforming finite elements, hence the name Finite Cell Method. Only cells intersecting the physical domain are considered during a Finite Cell simulation, which includes cells that lie completely in $\Omega_{\text {phys }}$ and the so-called cut cells that are intersected by the boundary of $\Omega_{\text {phys }}$. The solution space spanned by the shape functions is defined on the cells, while the original physical geometry $\Omega_{\text {phys }}$ is implicitly reconstructed by means of an indicator function $\alpha$ that associates an arbitrary point in $\Omega$ with either the physical or the fictitious domain. The indicator function $\alpha$ is defined as

$$
\alpha=\left\{\begin{array}{cl}
1 & \forall \mathbf{x} \in \Omega_{\text {phy }} \\
10^{-g} & \forall \mathbf{x} \in \Omega_{\text {fict }}
\end{array}\right.
$$

where, for numerical stability reasons $g$ is typically selected between 4 and 12. The discontinuity introduced by the indicator function $\alpha$ requires adaptive integration schemes. We refer to [33, 34] for further details. To numerically solve the problem discussed in Section 2 we employ a simple yet effective voxel-based integration scheme and refer the interested reader to [35] for further details.

The method is formulated such that high order basis functions can be directly employed to approximate the solution space. In particular, integrated Legendre polynomials and B-spline basis functions have already been extensively employed [36] 37.

Remark. Our implementation is completely general and can be used with any polynomial degree (and in the case of splines also with high continuity). However, we remark that, in the present work, for the sake of simplicity given the relatively simple geometries studied, we always adopt a polynomial degree $p=1$. Nevertheless, the possibility to increase it is surely attractive in particular when very complex geometries are involved (e.g., lattice structures) and/or when extremely accurate values of derived quantities such as residual strains and stresses 
are required. This specific topic will be studied in a forthcoming contribution.

Remark. In the presented implementation, the voxelized geometry is solely used to distribute the integration points in a simple way. The geometry of the part which is employed to perform the inside/outside test to evaluate the indicator function $\alpha$ and to impose the boundary conditions is the original .stl model. Therefore, this FCM discretization should not be confused with a voxel-based FEM approach (see, e.g., [38] ).

\subsection{Activation procedure}

Figure 5 shows the FCM cell discretization embedding the original stl data file together with the base plate support (cells completely outside the domain are not visualized). In all the performed analyses, we employ trilinear hexahedral elements for the approximation of the solution space, while on each integration voxel we distribute eight Gauss points. Since no conforming mesh generation is required, we can directly import the stl file into the numerical thermo-mechanical problem solver.

The presented methodology allows to distinguish between two different kind of layers:

1. cell-layer: the layer of finite cells where the basis functions are supported;

2. powder- or physical-layer: the layer corresponding to the actual layer of powder spread by the AM machine.

In the present implementation, at the beginning of each thermo-mechanical cycle a new cell-layer, including $m$ powder-layers, is created. As described in Section 4.1. the geometry is reconstructed only at the integration level by means of a voxel based integration grid, which is chosen to be much finer than the cell discretization. In particular, we use $4 \times 4 \times 4$ voxels in each finite cell and $(p+1)^{d}$ Gauss integration points in each voxel, where $p$ is the ansatz order of the basis functions and $d$ indicates the dimensionality of the problem. In case of trilinear finite cells for 3D problems, we have 8 Gauss points/voxel. 


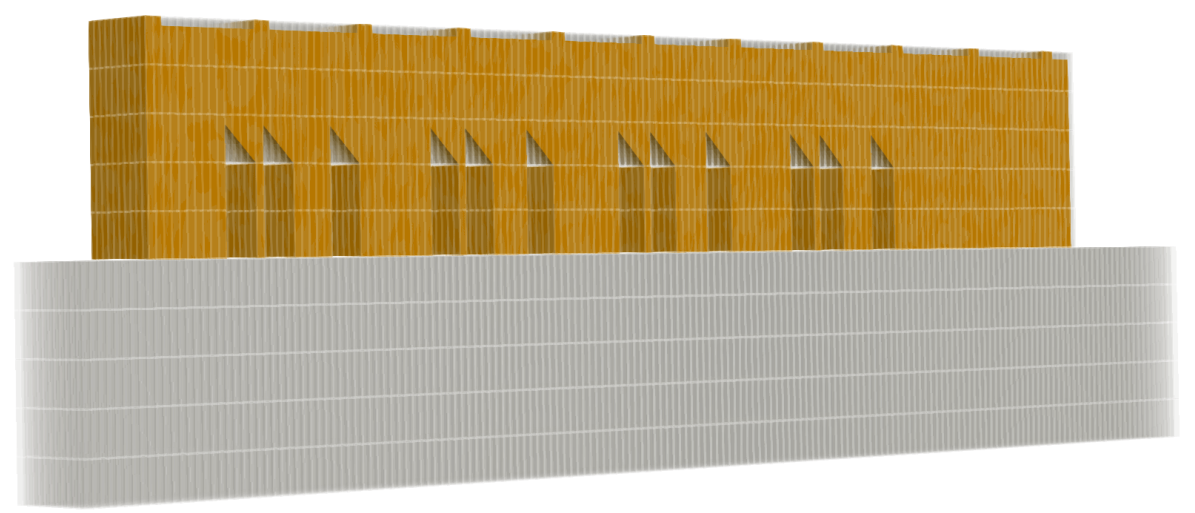

Figure 5: FCM cell edges (gray) embedding the original stl file (orange).

\subsection{Time integration}

In the presented numerical implementation, we employ a backward Euler implicit time integration scheme and we distinguish between a heating and a cooling time step having different time step sizes. Each time step can be further subdivided into smaller sub-steps to improve convergence. In a single heating time step, we activate a cell-layer $L$ including $m$ physical-layers.

The heating time step size $\Delta t_{h}^{L}$ depends on the heated surface (i.e., on the geometry of those specific powder layers). It is, thus, evaluated at run time as follows:

$$
\Delta t_{h}^{L}=\sum_{i=1}^{m} \frac{H A S_{i}}{h_{d} v}
$$

where $H A S_{i}$ is the heat affected surface of the $i^{t h}$-layer, $h_{d}$ the hatch distance of the specific laser scan strategy, and $v$ the laser velocity.

The cooling time step size $\Delta t_{c}^{L}$ is instead calculated as follows:

$$
\Delta t_{c}^{L}=m \bar{t}_{l}-\Delta t_{h}^{L}
$$

where $\bar{t}_{l}$ is the average layer time (see Table 1), i.e., the average time spent to print a single powder layer including the dwell time and the powder deposition 
time.

\subsection{Thermo-mechanical cycle}

Once a new cell-layer is generated, an inside-outside test is performed at each integration point (see Figure 6 to assign the value of the indicator function $\alpha$ defined in Section 4.1 At this stage of the process we are already able to start the thermo-mechanical cycle depicted in Figure 7

It is worth to point out that the total thickness of the $m$ new powder layers created within a single thermo-mechanical cycle does not have to correspond to the cell-layer height due to the immersed nature of the method. For instance, in Figure 6, the powder-layer thickness created in a cycle is half of the cell-layer height. Nevertheless, in both our numerical simulations and in the following description, at each thermo-mechanical cycle, we assume that the powder-layer thickness created in a cycle corresponds to the height of a single cell-layer. In particular, for the simulations presented in this work, we employ two different values of the parameter $m: m=125$ and $m=25$. Since each powder layer has a thickness of $20 \mu \mathrm{m}$, the corresponding cell thicknesses will be 2.5 and $0.5 \mathrm{~mm}$, respectively.

At the beginning of each thermo-mechanical cycle, a new cell-layer $L$ including $m$ powder-layers is activated and an equivalent thermal load $Q$ is applied onto the HAV of $L$. The HAV of a single cell-layer is evaluated as follows:

$$
\mathrm{HAV}=\sum_{i}^{n_{v}} V_{i}
$$

where $V_{i}$ is the volume of the $i^{\text {th }}$ voxel and $n_{v}$ is the total number of voxels in the cell $L$.

Equation (1) is then solved on the new domain for the heating time step increment $\Delta t_{h}^{L}$ and the evaluated temperature field is used to compute the thermal strain $\varepsilon^{t h}$ in the mechanical problem. The layer cycle is completed by a cooling time step where Equation (1) is solved for $Q=0$ and the residual stresses in the structure are generated. 


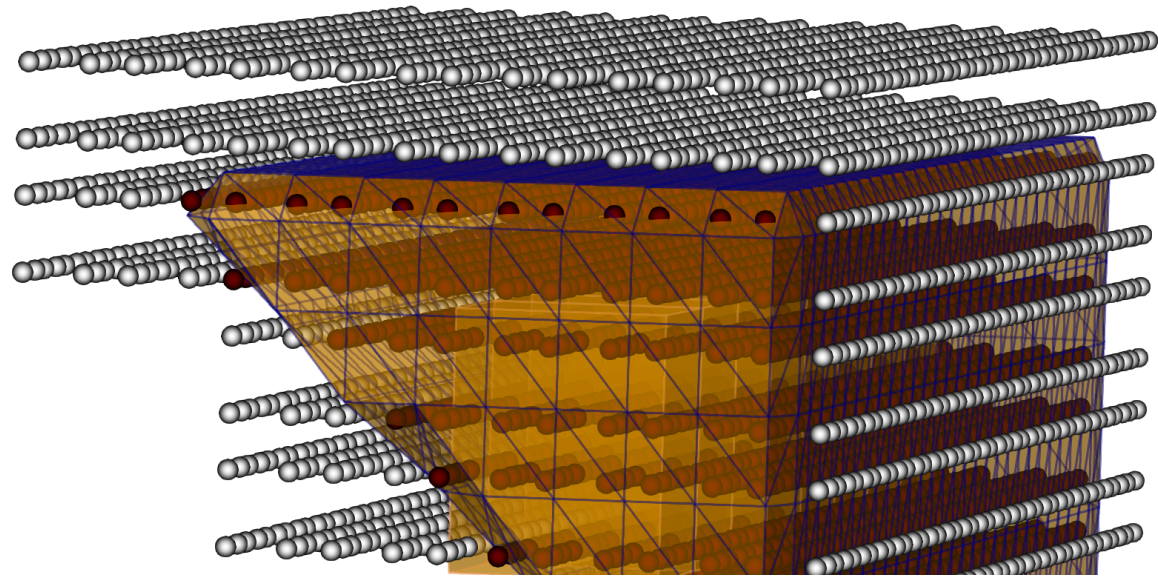

(a) Gauss integration points embedding the .stl growing domain.

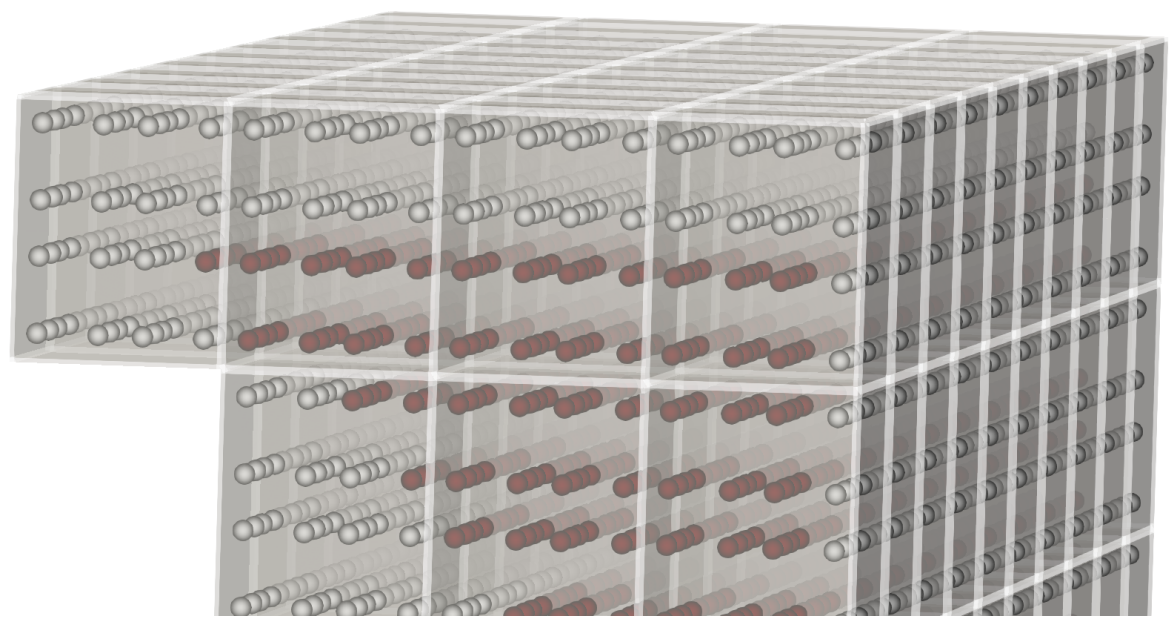

(b) Gauss integration points and the corresponding finite cell discretization.

Figure 6: Integration point inside-outside test. In red are represented the Gauss points lying within the physical domain, while in white are the Gauss points lying outside the domain. In this case, we employ a voxel-based integration grid with $2 \times 2 \times 2$ voxels/cell and $2^{3}$ Gauss points/voxel.

An important feature of the adopted physical model is the choice of the initial temperature of the newly created integration points of the mesh every time a new cell-layer is created. This value, in fact, is used to evaluate the thermal strain in the mechanical model and has a strong influence to predict the correct 


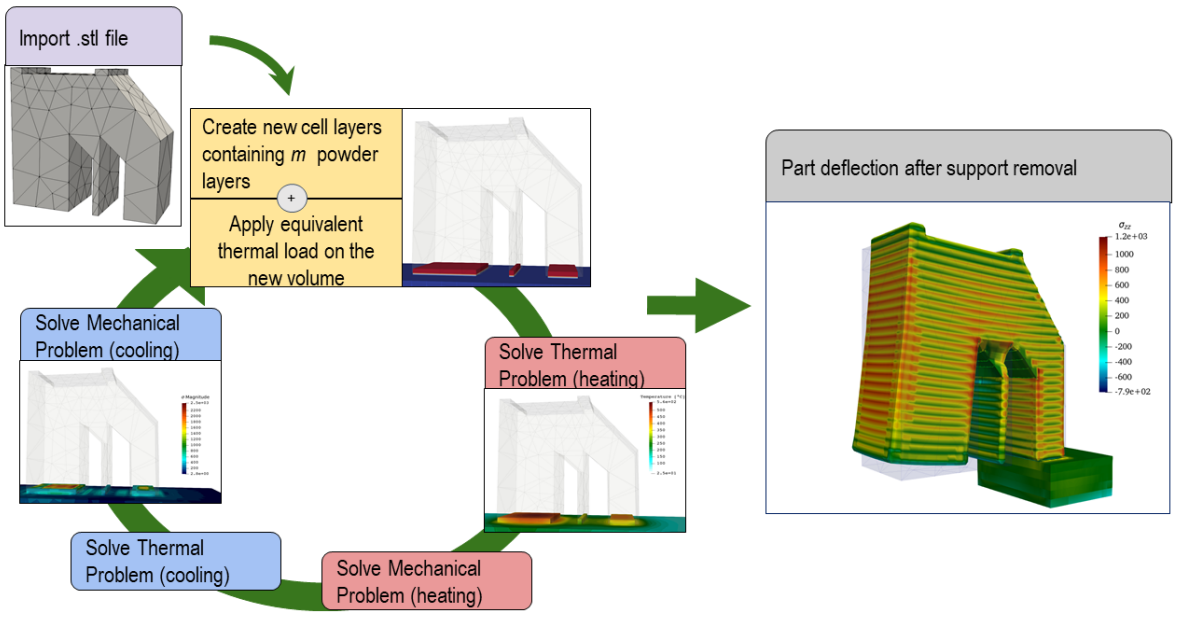

Figure 7: Thermo-mechanical part-scale modeling flowchart.

mechanical behavior during both the manufacturing process and the support removal stage. Following the pragmatic approach, we instantiate the new Gauss point temperature value at the so-called relaxation temperature defined as "the temperature above which thermal strain induces negligible thermal stress" [19], which for IN625 is $750^{\circ} \mathrm{C}$.

Once the entire LPBF process is simulated and the final residual stresses within the component are computed, a part-removal step is carried out where the base plate cells are progressively removed and the final deflection of the artifact is calculated.

The main difference between the proposed scheme and other similar multiplelayer activation schemes present in the literature is the possibility to separate the resolution of the geometry description from the solution approximation. Moreover, in this work the cells are newly generated as each layer is activated, i.e., we employ a so-called birth-death element procedure (Martukanitz et al. [39]). This choice is justified by the fact that generating a cell layer in a cartesian grid structure is an extremely simple operation. Thereby, about half the computational resources are saved during the course of the simulation and we can concentrate memory and computational power only where is actually needed, 
without allocating any space for inactive elements, as required for instance by the quiet element method [40].

\subsection{Material Properties}

The material considered in this work is the nickel-based superalloy IN625. This alloy is commonly used in industry and therefore its material properties are well-known and widely available. However, in AM applications, we need to obtain accurate measuments of material properties not only at ambient temperature but also at temperatures close to melting. The temperature-dependent material properties are taken from [41] and reported in Figure 8. In this work we implement a temperature independent yielding behavior with linear hardening as defined in 42 . 


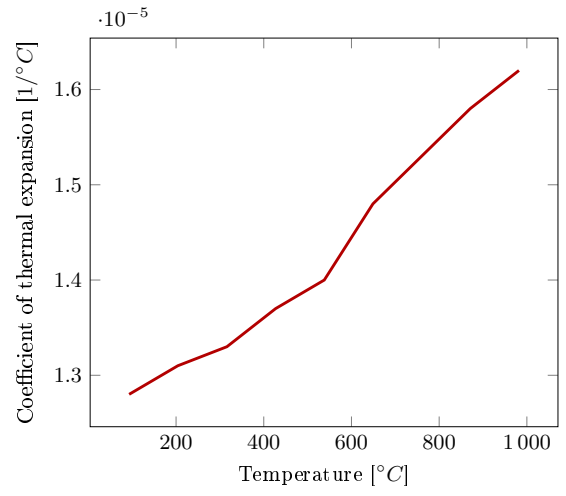

(a)

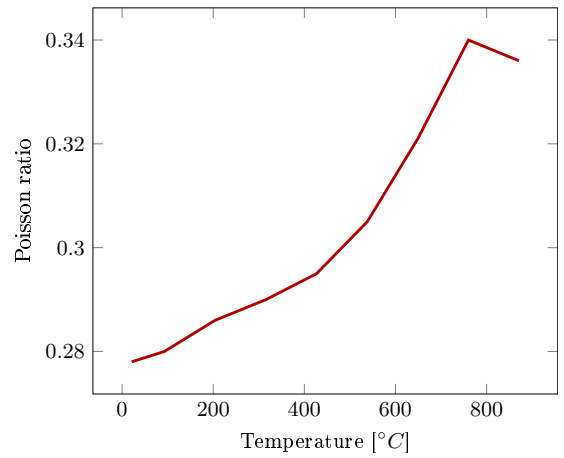

(c)

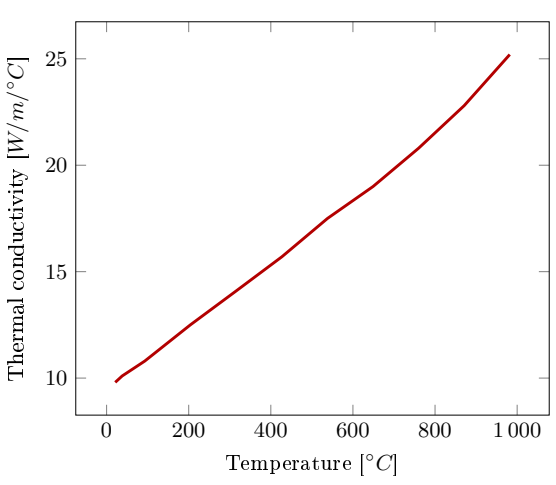

(e)

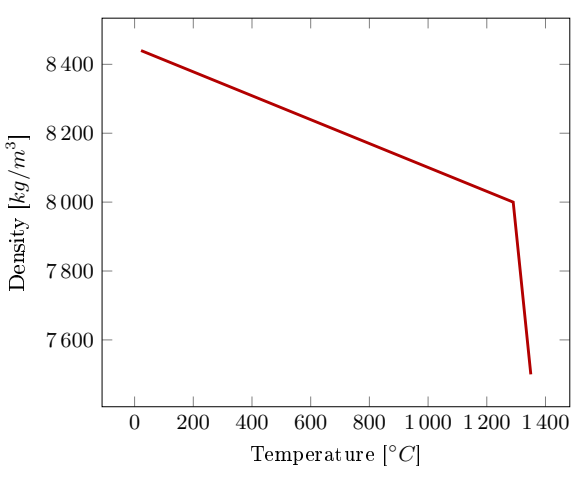

(b)

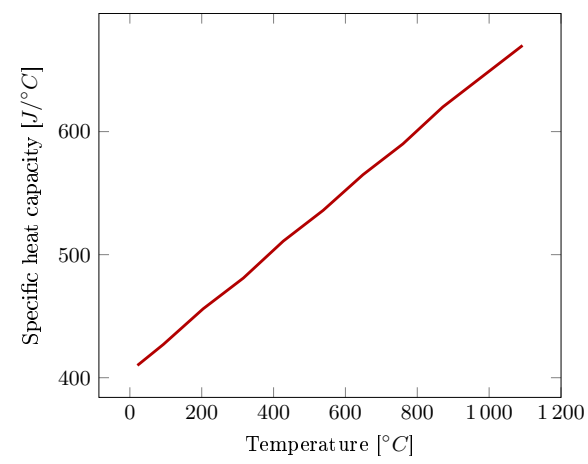

(d)

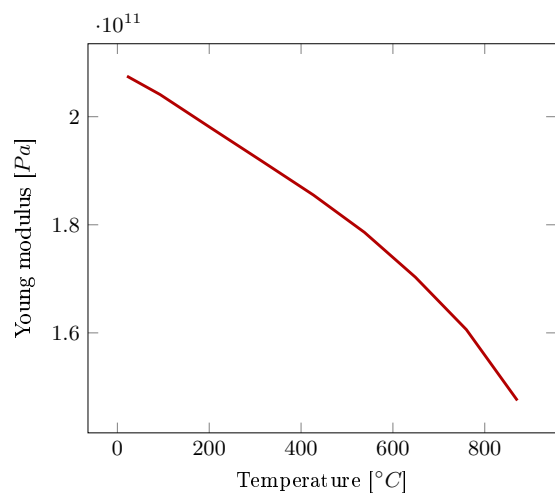

(f)

Figure 8: IN625: Temperature-dependent material properties (source 41]). 


\subsection{Domain discretization}

The original .stl file and the base plate domain are immersed within an embedded domain discretized using $180 \times 12 \times 2 N$ finite cells, where $N$ is the total number of cell-layers activated in the analysis. Since the total number of powder-layers of the structure is $625, N$ can be computed as

$$
N=\frac{625}{m}
$$

Therefore, choosing $m=125$, we have 5 elements in the structure height and 5 elements in the support height, and, for $m=25$, we have 25 elements in both the structure and the support height.

\subsection{Initial and Boundary conditions}

In our analysis, we assume that the temperature at the bottom of the base plate is constantly maintained at $80^{\circ} \mathrm{C}$, whereas the room temperature within the building chamber is $25^{\circ} \mathrm{C}$. Therefore, at the bottom of the base plate we apply a Dirichlet boundary condition (BC) imposing the temperature to be equal to the base plate temperature, while the initial temperature on the remaining degrees of freedom is set to the room temperature.

The heat dissipated by conduction through the powder and by convection and radiation through the upper surface of the structure is applied by means of Neumann BCs as described in Section 2.1. Mechanical boundary conditions are imposed clamping the bottom surface of the base plate and progressively removing base plate elements during the support removal stage.

A key feature of the presented implementation is that we can weakly apply both Dirichlet and Neumann boundary conditions directly on .stl geometry (see Figure 9). This feature turns out to be particularly important to correctly solve the thermal problem (see [43] for further details on the application of weak boundary conditions in FCM). 


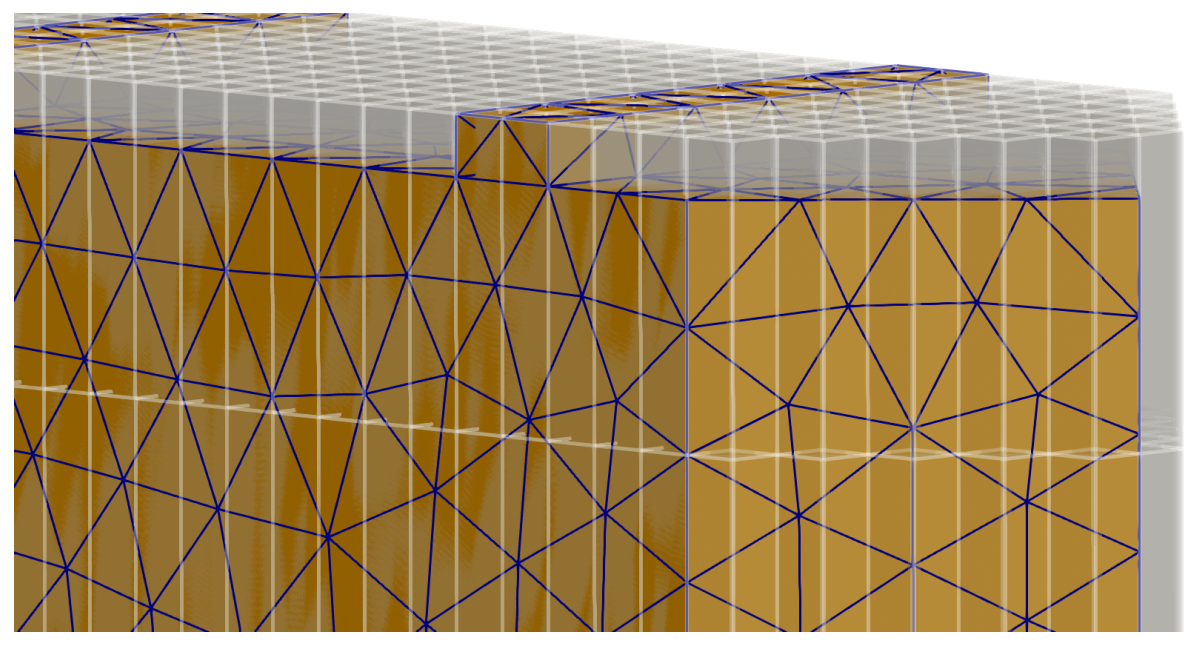

Figure 9: A geometrical detail of the triangular .stl surface (blue edges) used for the application of thermal boundary conditions. In gray the cells embedding the original domain. Note that the embedding domain is not matching the .stl surface boundaries.

\subsection{Error metrics}

In the following discussion, we employ two metrics to assess the accuracy of our numerical results with respect to experimental measurements:

1. The percent error of the maximum deflection (measured on the $11^{\text {th }}$ ridge of the structure, see Figure 31;

2. the correlation coefficient $C$ over vertical displacements at each ridge of the structure, defined as:

$$
\mathrm{C}(N, M) \%=\frac{\sum_{i=1}^{11}\left(u_{n, i}-\bar{u}_{n}\right)\left(u_{m, i}-\bar{u}_{m}\right)}{\sqrt{\sum_{i=1}^{11}\left(u_{n, i}-\bar{u}_{n}\right)^{2} \sum_{i=1}^{11}\left(u_{m, i}-\bar{u}_{m}\right)^{2}}} \times 100 \%
$$

where $M$ and $N$ are the set of measured and simulated data, $u_{m, i}$ and $u_{n, i}$ the corresponding displacement values of each set at the $i^{t h}$-ridge of the structure, and $\bar{u}_{m}$ and $\bar{u}_{n}$ are the corresponding mean values. Therefore, a correlation $C=100 \%$ indicates a perfect correlation between the numerical results and the experimental measurements. 


\begin{tabular}{c|c|c|c}
$\mathrm{X}$ & Measured & $m=125$ & $m=25$ \\
\hline 0.5 & 1.276 & 1.33 & 1.256 \\
7.5 & 0.997 & 1 & 0.98 \\
14.5 & 0.754 & 0.76 & 0.73 \\
21.5 & 0.551 & 0.56 & 0.53 \\
28.5 & 0.387 & 0.4 & 0.37 \\
35.5 & 0.25 & 0.26 & 0.24 \\
42.5 & 0.146 & 0.15 & 0.15 \\
49.5 & 0.065 & 0.06 & 0.07 \\
56.5 & 0.012 & 0.01 & 0.02 \\
63.5 & 0 & -0.02 & 0 \\
70.5 & 0.003 & -0.02 & -0.01 \\
\hline
\end{tabular}

Table 2: Experimental measurements and numerical results of upward deflection at the midpoint of each ridge $(\mathrm{X})$.

\section{Results and discussion}

All the numerical analyses discussed in this section have been obtained using AdhoC++ on a desktop computer provided with Intel ${ }^{\circledR}$ Core $^{\text {TM }}$ i7-6700, CPU@4.0GHz, RAM 64Gb.

The approximated upward deflections measured at the mid-point of each grounded ridge are reported in Table 2 and Figure 10 together with the corresponding simulated results. Table 3 reports the relative maximum deflection error percentage, the correlation value $C$, and the total wall-clock time of the simulated results.

The coarse analysis $(m=125)$ delivers an error on the maximum deflection of $4.72 \%$, while in the refined analysis $(m=25)$ this error drops down to approximately $1 \%$. Both analyses show an excellent (almost perfect) correlation with respect to the measured data. The computational speed up obtained by means of the coarse analysis is very significant, dropping from more than 8 hours to less than 90 minutes (factor 5.5). Moreover, the computational time can be consistently further reduced employing a distributed parallel implementation, which will be addressed in a forthcoming contribution.

Figure 11 shows the temperature distribution at each heating time step (i.e., when the equivalent heat source is applied in the new layer), on the embedded 


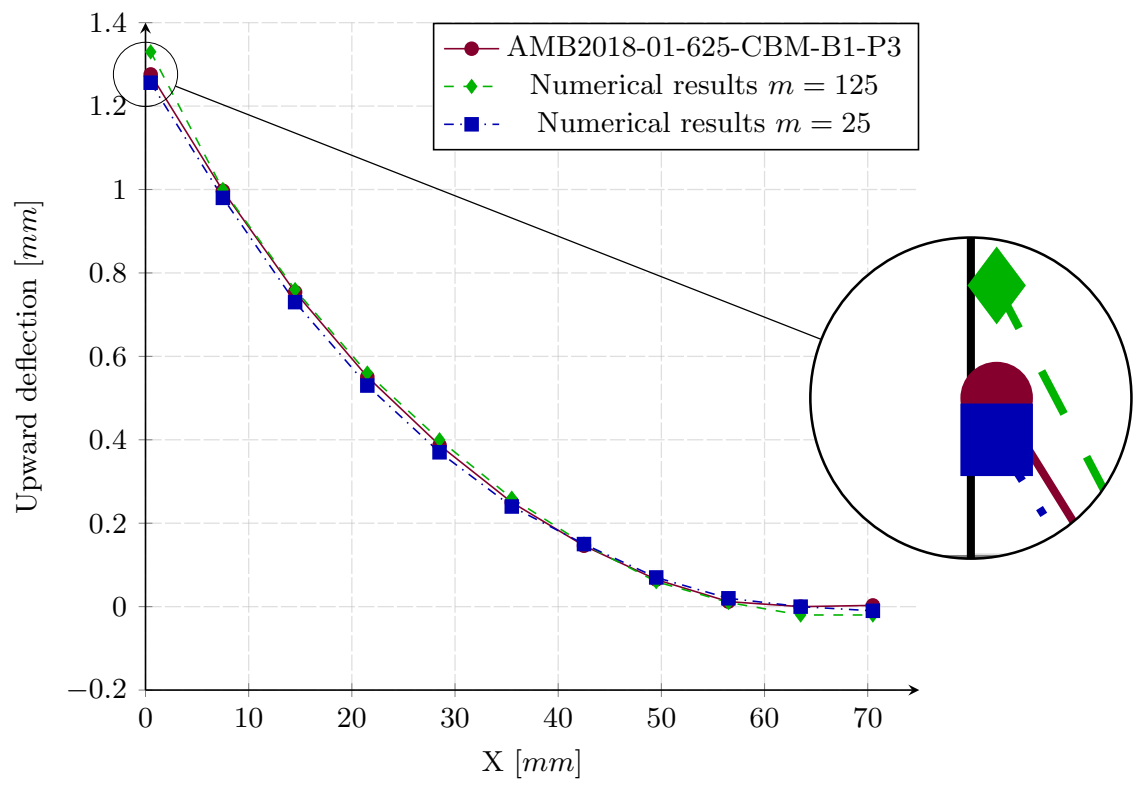

Figure 10: Part deflection measured at each ridge of the cantilever structure.

\begin{tabular}{c|c|c|c} 
& max. error [\%] & $C[\%]$ & Wall-clock time \\
\hline$m=125$ & 4.72 & 99.98 & $1 \mathrm{~h} 28 \mathrm{~m}$ \\
\hline$m=25$ & 1.10 & 99.99 & $8 \mathrm{~h} 19 \mathrm{~m}$ \\
\hline
\end{tabular}

Table 3: Maximum deflection error, correlation coefficient, and total wall-clock time of the numerical simulations.

.stl surface geometry for the case $m=125$ (our coarsest discretization). The same surface geometry is also used in our implementation to apply thermal boundary conditions.

Figure 12 and Figure 13 show the von Mises stress distribution at the end of the last cooling time step for the cases $m=125$ and $m=25$, respectively. During support removal, residual stresses are relieved generating an upward deflection of the final structure. In Figure 12 we can observe that in the building direction the stresses are characterized by stronger jumps at the element interfaces compared to the results of Figure 13 . This effect is due to the coarser cell size employed for the analysis along the building direction. Nevertheless, 


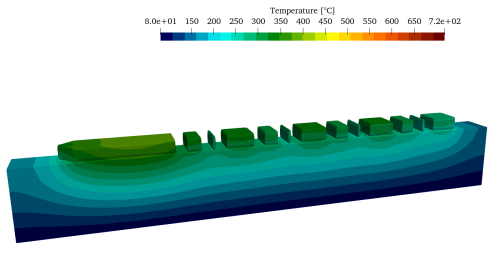

(a)

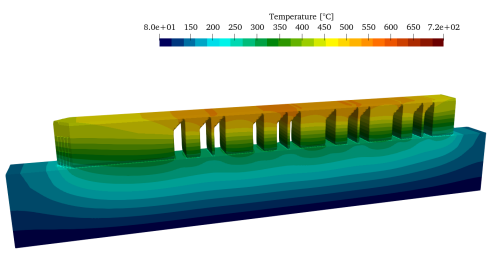

(c)

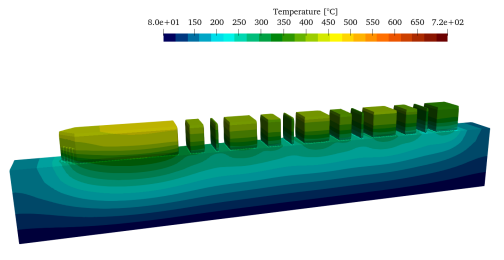

(b)

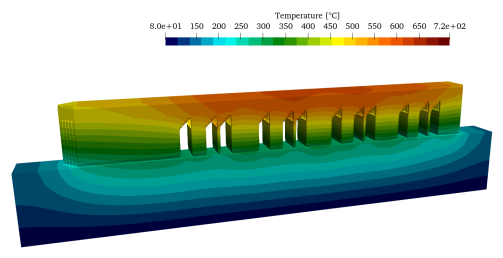

(d)

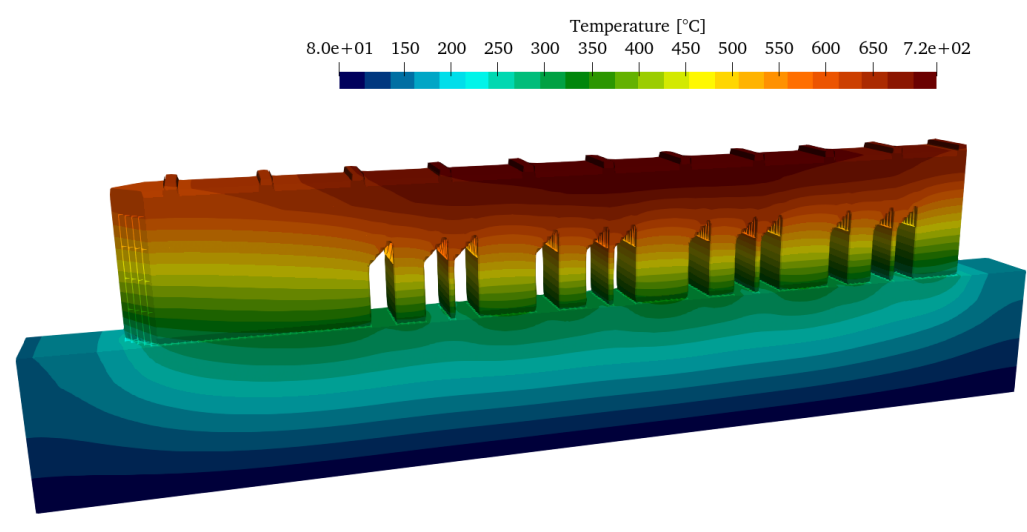

(e)

Figure 11: Temperature distribution at each heating time step.

the accuracy of the predicted part deflection is not drastically affected and the relative error on the maximal deflection is kept below $5 \%$.

A possible interpretation of these results starts from the observation that in both computations the geometry is represented with the same accuracy. Therefore, the only differences between the two analyses is the number of thermo- 


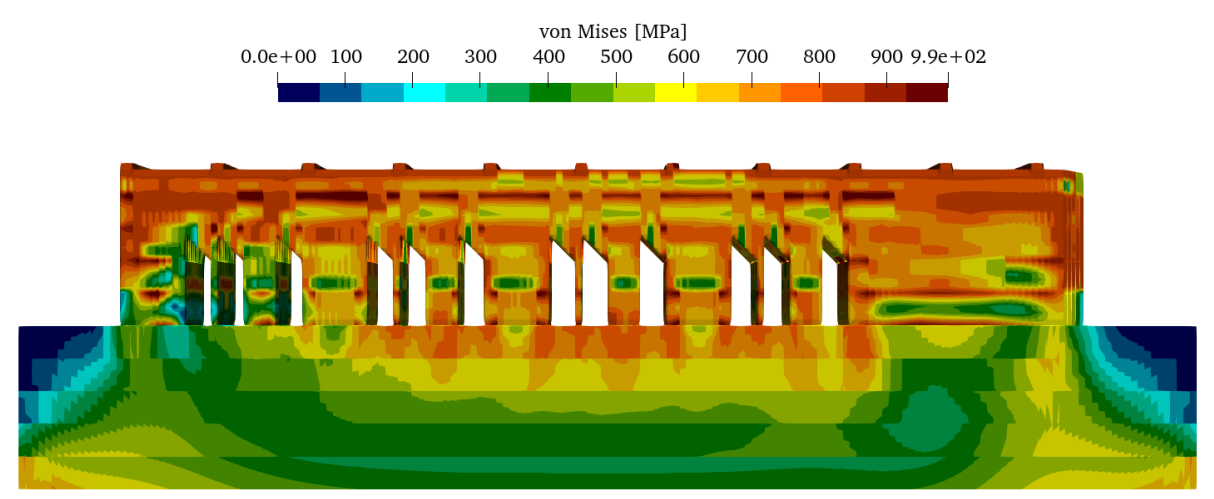

Figure 12: Residual von Mises stresses at the end of the process $(m=125)$.

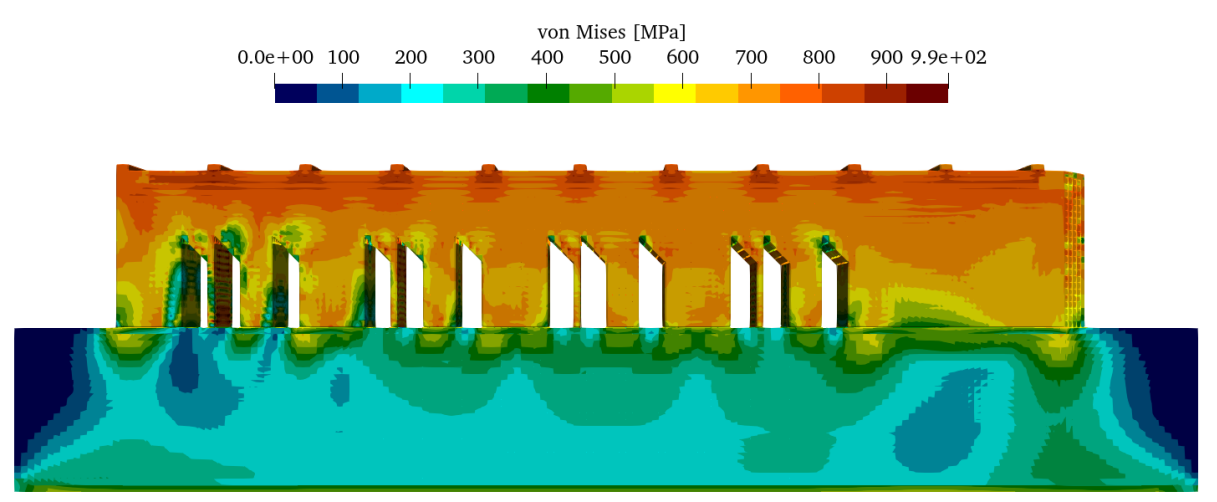

Figure 13: Residual von Mises stresses at the end of the process $(m=25)$.

mechanical cycles (5 and 25, respectively) and the number of finite cells in the growing direction (10 and 50, respectively). Since the layer thickness of a single powder-layer is much smaller than in the other two dimensions, shear stresses induced by the newly deposited layers on the previous layers are quite small compared to in-plane, normal stresses; thus a coarse mesh in the building direction is still able to well capture part-deflection induced by residual stresses.

Employing an immersed approach, i.e., decoupling the geometry representation from the discretization of the solution space, allows to adopt very coarse elements along the building direction paying a negligible price in terms of accuracy. On the contrary, a standard FEM approach using such a coarse dis- 
cretization along the growing direction would lead to less accurate results since the geometry cannot be represented with sufficient accuracy anymore. Clearly, for the present benchmark numerical simulations can be obtained also by means of classical FEM codes, e.g., in Yang et al. [19] an excellent agreement with the measured stains of the final structure is obtained using ABAQUS AM ${ }^{\circledR}$.

However, the main advantage of the proposed immersed boundary workflow compared to conforming mesh discretization lies in the possibility to perform design-through-analysis within a single, yet effective numerical environment and without the necessity to generate finite element meshes which conform to the boundary of the simulated artifact.

\section{Conslusions}

Obtaining a suitable FEM discretization from complex CAD models can be very challenging and time-consuming. By means of the Finite Cell Method, we are able to implicitly reconstruct the geometry of the produced part without generating a conforming mesh. Combining FCM and a simple yet effective partscale model, we are able to accurately capture experimental measurements of part deflection after support removal.

The immersed nature of FCM allows to employ a very coarse discretization, able to perform the entire calculation in less than 90 minutes on a standard desktop computer, delivering at the same time results with a very accurate correlation with experimental measurements while keeping the maximum deflection error below 5\%. Employing a finer discretization leads to very similar results in terms of correlation percentage, while the maximum deflection error decreases down to approximately $1 \%$.

It is concluded that the use of an immersed methodology to perform LPBF large scale process simulations opens the possibility to rapidly estimate part deformation directly within a CAD environment, since there is no need of a conforming mesh generation process. This aspect, which is generally neglected in most of the literature on LPBF simulation, could instead be crucial in the 
industrial practice.

Possible further developments of the present work can include an extension of the numerical scheme to allow local refinement and the use of a more sophisticated multi-scale physical model, such as the modified inherent strain method [16]. Moreover, the computational time could be further reduced by means of parallel computation and code parallelization.

\section{Acknowledgments}

The authors gratefully acknowledge the Financial support of the German Research Foundation (DFG) under Grant RA 624/27-2. This work was partially supported by Regione Lombardia through the project "TPro.SL - Tech Profiles for Smart Living" (No. 379384) within the Smart Living program, and through the project "MADE4LO - Metal ADditivE for LOmbardy" (No. 240963) within the POR FESR 2014-2020 program and by the MIUR-PRIN project XFAST-SIMS (no. 20173C478N). John Jomo is grateful for the support of the International Graduate School of Science and Engineering at the Technical University of Munich for funding this research.

\section{References}

[1] W. E. King, A. T. Anderson, R. M. Ferencz, N. E. Hodge, C. Kamath, S. A. Khairallah, A. M. Rubenchik, Laser powder bed fusion additive manufacturing of metals; physics, computational, and materials challenges, Applied Physics Reviews 2 (2015).

[2] M. Galati, L. Iuliano, A literature review of powder-based electron beam melting focusing on numerical simulations, Additive Manufacturing 19 (2018) 1 - 20. URL: http://www.sciencedirect. com/science/article/pii/S2214860417300635, doi:https://doi.org/ $10.1016 / \mathrm{j}$. addma. 2017.11 .001 . 
[3] Z. Yan, W. Liu, Z. Tang, X. Liu, N. Zhang, M. Li, H. Zhang, Review on thermal analysis in laser-based additive manufacturing, Optics \& Laser Technology 106 (2018) 427 - 441. URL: http://www.sciencedirect. com/science/article/pii/S0030399217317140, doi/https://doi.org/ $10.1016 / \mathrm{j}$. optlastec. 2018.04 .034

[4] B. Schoinochoritis, D. Chantzis, K. Salonitis, Simulation of metallic powder bed additive manufacturing processes with the finite element method: A critical review, Proceedings of the Institution of Mechanical Engineers, Part B: Journal of Engineering Manufacture 231 (2017) 96-117. doi:10. $1177 / 0954405414567522$.

[5] W. King, A. T. Anderson, R. M. Ferencz, N. E. Hodge, C. Kamath, S. A. Khairallah, Overview of modelling and simulation of metal powder bed fusion process at lawrence livermore national laboratory, Materials Science and Technology 31 (2015) 957-968.

[6] S. A. Khairallah, A. T. Anderson, A. Rubenchik, W. E. King, Laser powder-bed fusion additive manufacturing: Physics of complex melt flow and formation mechanisms of pores, spatter, and denudation zones, Acta Materialia 108 (2016) 36-45. doi:10.1016/j .actamat.2016.02.014

[7] C. Körner, E. Attar, P. Heinl, Mesoscopic simulation of selective beam melting processes, Journal of Materials Processing Technology 211 (2011) 978-987. doi:10.1016/j.jmatprotec.2010.12.016.

[8] S. Sahoo, K. Chou, Phase-field simulation of microstructure evolution of Ti-6Al-4V in electron beam additive manufacturing process, Additive Manufacturing 9 (2016) 14 - 24. URL: http://www. sciencedirect. com/science/article/pii/S2214860415300051. doi:https://doi.org/ $10.1016 / \mathrm{j}$. addma. 2015.12 .005 .

[9] A. Rai, M. Markl, C. Körner, A coupled cellular AutomatonLattice Boltzmann model for grain structure simulation during additive manufacturing, Computational Materials Science 124 (2016) 
37 - 48. URL: http://www.sciencedirect.com/science/article/ pii/S092702561630324X doi/https://doi.org/10.1016/j.commatsci. 2016.07 .005 .

[10] E. Salsi, M. Chiumenti, M. Cervera, Modeling of microstructure evolution of Ti6Al4V for additive manufacturing, Metals 633 (2018).

[11] E. R. Denlinger, J. Irwin, P. Michaleris, Thermomechanical Modeling of Additive Manufacturing Large Parts, Journal of Manufacturing Science and Engineering 136 (2014) 061007. (8 pages).

[12] M. Chiumenti, X. Lin, M. Cervera, W. Lei, Y. Zheng, W. Huang, Numerical simulation and experimental calibration of additive manufacturing by blown powder technology. Part I: Thermal analysis, Rapid Prototyping Journal 23 (2017) 448-463. doi:10.1108/RPJ-10-2015-0136

[13] L.-E. Lindgren, A. Lundbäck, M. Fisk, R. Pederson, J. Andersson, Simulation of additive manufacturing using coupled constitutive and microstructure models, Additive Manufacturing 12 (2016) 144-158.

[14] D. Soldner, J. Mergheim, Thermal modelling of selective beam melting processes using heterogeneous time step sizes, Computers \& Mathematics with Applications 78 (2019) 2183 - 2196. URL: http://www.sciencedirect. com/science/article/pii/S0898122118302505, doi:https://doi.org/ 10.1016/j.camwa.2018.04.036, simulation for Additive Manufacturing.

[15] M. Gouge, E. Denlinger, J. Irwin, C. Li, P. Michaleris, Experimental validation of thermo-mechanical part-scale modeling for laser powder bed fusion processes, Additive Manufacturing 29 (2019) 100771. URL: http://www.sciencedirect.com/science/article/pii/ S2214860418310340. doi:https://doi.org/10.1016/j.addma.2019.06. 022 .

[16] X. Liang, Q. Chen, L. Cheng, D. Hayduke, A. C. To, Modified inherent strain method for efficient prediction of residual deformation in direct metal 
laser sintered components, Computational Mechanics 64 (2019) 1719-

1733. URL: https://doi.org/10.1007/s00466-019-01748-6, doi:10. 1007/s00466-019-01748-6.

[17] X. Liang, L. Cheng, Q. Chen, Q. Yang, A. C. To, A modified method for estimating inherent strains from detailed process simulation for fast residual distortion prediction of single-walled structures fabricated by directed energy deposition, Additive Manufacturing 23 (2018) 471 - 486. URL: http://wWw.sciencedirect.com/science/article/pii/ S2214860418304858, doi/https://doi.org/10.1016/j .addma.2018.08. 029.

[18] R. J. Williams, C. M. Davies, P. A. Hooper, A pragmatic part scale model for residual stress and distortion prediction in powder bed fusion, Additive Manufacturing 22 (2018) 416 - 425. URL: http://www.sciencedirect. com/science/article/pii/S2214860418300514, doi/https://doi.org/ $10.1016 / \mathrm{j}$. addma.2018.05.038.

[19] Y. Yang, M. Allen, T. London, V. Oancea, Residual strain predictions for a powder bed fusion inconel 625 single cantilever part, Integrating Materials and Manufacturing Innovation 8 (2019) 294-304. doi:10.1007/ s40192-019-00144-5

[20] J. Cottrell, T. J. R. Hughes, Y. Bazilevs, Isogeometric Analysis: Toward Integration of CAD and FEA, Wiley and Sons, 2009.

[21] I. Gibson, D. W. Rosen, B. Stucker, Additive Manufacturing Technologies, Springer-Verlag New York, 2015.

[22] J. Parvizian, A. Düster, E. Rank, Finite cell method, Computational Mechanics 41 (2007) 121-133. doi:10.1007/s00466-007-0173-y.

[23] S. Kollmannsberger, A. Özcan, M. Carraturo, N. Zander, E. Rank, A hierarchical computational model for moving thermal loads and phase changes with applications to selective laser melting, Computers \& Mathematics 
with Applications 75 (2018) 1483-1497. doi:10.1016/j.camwa.2017.11. 014.

[24] A. Özcan, S. Kollmannsberger, J. Jomo, E. Rank, Residual stresses in metal deposition modeling: Discretizations of higher order, Computers \& Mathematics with Applications 78 (2019) 2247 - 2266. doi:https://doi. org/10.1016/j.camwa.2018.10.027

[25] J. A. Goldak, M. Akhlaghi, Computational Welding Mechanics, Springer, New York, 2005.

[26] L.-E. Lindgren, Numerical modelling of welding, Computer Methods in Applied Mechanics and Engineering 195 (2006) 6710-6736.

[27] M. Gauge, P. Michaleris, Thermo-Mechanical Modeling of Additive Manufacturing, Butterworth-Heinemann, 2017.

[28] M. Chiumenti, E. Neiva, E. Salsi, M. Cervera, S. Badia, J. Moya, Z. Chen, C. Lee, C. Davies, Numerical modelling and experimental validation in Selective Laser Melting, Additive Manufacturing 18 (2017) 171-185. doi: $10.1016 / \mathrm{j}$. addma. 2017.09 .002 .

[29] www.nist.gov/ambench, AM Bench benchmark challenge CHALAMB2018-02-MP, $\quad$ https://www.nist.gov/ambench/amb2018-02description, 2018.

[30] T. Q. Phan, M. Strantza, M. R. Hill, T. H. Gnaupel-Herold, J. Heigel, C. R. D'Elia, A. T. DeWald, B. Clausen, D. C. Pagan, J. Y. P. Ko, D. W. Brown, L. E. Levine, Elastic Residual Strain and Stress Measurements and Corresponding Part Deflections of 3D Additive Manufacturing Builds of IN625 AM-Bench Artifacts Using Neutron Diffraction, Synchrotron X-Ray Diffraction, and Contour Method, Integrating Materials and Manufacturing Innovation (2019).

[31] A. Özcan, S. Kollmannsberger, J. Jomo, E. Rank, Residual stresses in metal deposition modeling: discretizations of higher order, Computers 
\& Mathematics with Applications (2018). doi:10.1016/j.camwa.2018.10. 027.

[32] A. Düster, J. Parvizian, Z. Yang, E. Rank, The finite cell method for three-dimensional problems of solid mechanics, Computer Methods in Applied Mechanics and Engineering 197 (2008) 3768-3782. doi:10.1016/j . Cma.2008.02.036.

[33] A. Abedian, J. Parvizian, A. Düster, H. Khademyzadeh, E. Rank, Performance of Different Integration Schemes in Facing Discontinuities in the Finite Cell Method, International Journal of Computational Methods 10 (2013) 1350002. doi:10.1142/S0219876213500023, 00027.

[34] L. Kudela, N. Zander, S. Kollmannsberger, E. Rank, Smart octrees: Accurately integrating discontinuous functions in 3D, Computer Methods in Applied Mechanics and Engineering 306 (2016) 406-426. doi:10.1016/j. cma.2016.04.006.

[35] Z. Yang, S. Kollmannsberger, A. Düster, M. Ruess, E. G. Garcia, R. Burgkart, E. Rank, Non-standard bone simulation: Interactive numerical analysis by computational steering, Computing and Visualization in Science 14 (2012) 207-216. doi:10.1007/s00791-012-0175-y.

[36] D. D'Angella, S. Kollmannsberger, E. Rank, A. Reali, Multi-level Bézier extraction for hierarchical local refinement of Isogeometric Analysis, Computer Methods in Applied Mechanics and Engineering 328 (2018) 147-174. doi: $10.1016 /$ j.cma.2017.08.017.

[37] D. Schillinger, M. Ruess, N. Zander, Y. Bazilevs, A. Düster, E. Rank, Small and large deformation analysis with the p- and B-spline versions of the Finite Cell Method, Computational Mechanics 50 (2012) 445-478. doi $10.1007 / \mathrm{s} 00466-012-0684-\mathrm{z}$.

[38] N. Keller, F. Neugebauer, H. Xu, V. Ploshikhin, Thermo-mechanical sim- 
ulation of additive layer manufacturing of titanium aerospace structures, in: Proceedings of the LightMAT Conference, 2013.

[39] R. Martukanitz, P. Michaleris, T. Palmer, T. DebRoy, Z.-K. Liu, R. Otis, T. W. Heo, L.-Q. Chen, Toward an integrated computational system for describing the additive manufacturing process for metallic materials, Additive Manufacturing 1-4 (2014) 52-63. doi:10.1016/j .addma.2014.09.002

[40] P. Michaleris, Modeling metal deposition in heat transfer analyses of additive manufacturing processes, Finite Elements in Analysis and Design 86 (2014) 51-60. doi:10.1016/j.finel.2014.04.003, 00006.

[41] www.specialmetals.com, INCONEL 625 Material Properties, http://www.specialmetals.com, 2019.

[42] EOS, EOS nickelalloy IN625, http://www.eos.info, 2019.

[43] S. Kollmannsberger, A. Özcan, J. Baiges, M. Ruess, E. Rank, A. Reali, Parameter-free, weak imposition of Dirichlet boundary conditions and coupling of trimmed and non-conforming patches, International Journal for Numerical Methods in Engineering 101 (2015) 670-699. doi:10.1002/nme. 4817, 00001. 